\title{
Long-segment posterior cervical decompression and fusion: does caudal level affect revision rate?
}

\author{
Kevin Hines, MD, ${ }^{1}$ Zachary T. Wilt, MD, ${ }^{2}$ Daniel Franco, MD, ${ }^{1}$ Aria Mahtabfar, MD,, Nicholas Elmer, ${ }^{1}$ \\ Glenn A. Gonzalez, MD, ${ }^{1}$ Thiago S. Montenegro, MD,, Lohit Velagapudi, ${ }^{1}$ Parthik D. Patel, MD, ${ }^{2}$ \\ Maxwell Detweiler, ${ }^{1}$ Umma Fatema, ${ }^{1}$ Gregory D. Schroeder, MD, ${ }^{2}$ and James Harrop, MD ${ }^{1}$
}

\begin{abstract}
'Department of Neurosurgery, Thomas Jefferson University and Jefferson Hospital for Neuroscience, Philadelphia; and ${ }^{2}$ Department of Orthopaedic Surgery, Rothman Institute, Thomas Jefferson University, Philadelphia, Pennsylvania
\end{abstract}

\begin{abstract}
OBJECTIVE Posterior cervical decompression and fusion (PCDF) is a commonly performed procedure to address cervical myelopathy. A significant number of these patients require revision surgery for adjacent-segment disease (ASD) or pseudarthrosis. Currently, there is no consensus among spine surgeons on the inclusion of proximal thoracic spine instrumentation. This study investigates the benefits of thoracic extension in long-segment cervical fusions and the potential drawbacks. The authors compare outcomes in long-segment subaxial cervical fusion for degenerative cervical myelopathy with caudal vertebral levels of $\mathrm{C} 6, \mathrm{C} 7$, and $\mathrm{T} 1$.
\end{abstract}

METHODS A retrospective analysis identified 369 patients who underwent PCDF. Patients were grouped by caudal fusion level. Reoperation rates for ASD and pseudarthrosis, infection, and blood loss were examined. Data were analyzed with chi-square, 1-way ANOVA, and logistic regression.

RESULTS The total reoperation rate for symptomatic pseudarthrosis or ASD was $4.8 \%$. Reoperation rates, although not significant, were lower in the C3-6 group (2.6\%, vs $8.3 \%$ for C3-7 and 3.8\% for C3-T1; $p=0.129)$. Similarly, rates of infection were lower in the shorter-segment fusion without achieving statistical significance $(2.6 \%$ for C3-6, vs $5.6 \%$ for C3-7 and 5.5\% for C3-T1; $p=0.573$ ). The mean blood loss was documented as 104, 125, and $224 \mathrm{~mL}$ for groups 1, 2, and 3 , respectively $(p<0.001)$.

CONCLUSIONS Given the lack of statistical difference in reoperation rates for long-segment cervical fusions ending at C6, C7, or T1, shorter fusions in high-risk surgical candidates or elderly patients may be performed without higher rates of reoperation.

https://thejns.org/doi/abs/10.3171/2020.10.SPINE201385

KEYWORDS cervical myelopathy; adjacent-segment disease; pseudarthrosis; revision surgery; posterior cervical fusion; degenerative

$\mathrm{P}$ OSTERIOR cervical decompression and fusion (PCDF) is a common procedure performed to address many pathologies such as cervical myelopathy, trauma, infection, and neoplasm. In cervical myelopathy, multilevel decompression and fusion is often required to address diffuse degenerative pathology. ${ }^{1}$ The annual rate of PCDF done in the United States has increased 2.7- and 2.9-fold since 2001 for degenerative disease and myelopathy, respectively, outcomes have been proven to be positive, and the surgical procedure is very well tolerated..$^{2-4}$ However, a significant number of these patients require further surgery as they develop adjacent-segment disease (ASD) caudal to the level of fusion. ${ }^{5}$ Rates of ASD requiring reoperation occur in as high as $27.8 \%$ of patients undergoing
PCDF. ${ }^{6}$ In addition to ASD, some patients fail to achieve fusion - and develop pseudarthrosis in $21.62 \%$ of cases.?

It has been suggested that ending long-segment fusions such as these in the rigid thoracic spine may provide more support and require less revision for ASD and pseudarthrosis $^{6-8}$ than for constructs ending at the transitional biomechanical zone in the cervicothoracic junction. Postoperative complications such as neck pain and infection can occur because there is extensive muscle detachment and retraction needed to perform a $\mathrm{PCDF}^{4}{ }^{4} \mathrm{C} 5$ palsy is also a known issue that occurs in approximately $6 \%-9 \%$ of cases, as well as potentially transient neurological worsening after extensive decompression. ${ }^{9}$ Some studies have advocated that healthy cervicothoracic junctions do not

ABBREVIATIONS ASD = adjacent-segment disease; $L O S=$ length of stay; PCDF = posterior cervical decompression and fusion.

SUBMITTED July 26, 2020. ACCEPTED October 12, 2020.

INCLUDE WHEN CITING Published online April 23, 2021; DOI: 10.3171/2020.10.SPINE201385. 
break down at an increased rate and do not require extension to the thoracic spine. ${ }^{10}$ Other risk factors previously associated with ASD include surgery at a young age, high T1 slope, and disruption of adjacent soft tissue..11,12 Currently there is no consensus among spine surgeons on the inclusion of proximal thoracic spine instrumentation for the purpose of decreasing revision rates. Some studies suggest that there is a major increase in fusion rates if the construct is extended down to $\mathrm{T} 1$-however, there seem to be discrepancies in this evidence throughout the available literature. ${ }^{9,13-15}$ The length of the construct has not been reliably found to be associated with the incidence of ASD or pseudarthrosis cases.

In this study, we hope to clarify the benefits of thoracic extension in long-segment cervical fusions and to evaluate potential drawbacks. If longer fusions do decrease breakdown rates, it is important to assess potential loss of range of motion, higher infection rate, longer surgeries, and higher blood loss. To develop practice recommendations, it is important to clarify the benefit of what would be a more invasive surgery with potentially higher morbidity. Given the limited data available, we compare outcomes in long-segment subaxial cervical fusion for degenerative cervical myelopathy with caudal vertebral levels of C6, $\mathrm{C} 7$, and $\mathrm{T} 1$.

\section{Methods}

This study was approved by our institutional review board. Given the retrospective nature of the study without effect on treatment or outcome, patient consent was not required. After approval, a database of cervical spine surgeries performed at a single center by spine surgeons was generated by searching for patients with ICD codes M48.02 (spinal stenosis, cervical region) and M47.12 (other spondylosis with myelopathy, cervical region) and instrumentation code 22842 (posterior segmental instrumentation). Patients were included if they underwent posterior subaxial decompression and fusion between 2010 and 2019, minimum fusion of 3 levels, and were documented as having signs and symptoms of myelopathy on preoperative evaluation. Patients with other indications or presentations such as tumor, infection, and trauma were excluded. Patients were also excluded if they had prior cervical surgery or combination procedures with anterior support. Patients were grouped by fusion levels C3-6 (group 1), C3-7 (group 2), and C3-T1 (group 3). Data on reoperation for symptomatic ASD or pseudarthrosis were collected, as were data on infection requiring wound revision, blood loss, length of stay (LOS), caudal level of decompression, and follow-up when available. Data on patient age, tobacco use at time of surgery, and use of biological material were also gathered for logistic regression.

In this study, ASD was defined as radiographic stenosis adjacent to the construct in symptomatic patients clinically requiring revision to further decompress and extend the fusion construct across the level of ASD as determined by the surgeon. Similarly, pseudarthrosis was defined as the radiographic loosening or failure of hardware and lack of fusion across a joint in symptomatic patients clinically requiring revision surgery to achieve arthrodesis and stability as determined by the surgeon.

\section{Statistical Analysis}

All statistical analyses were performed using SPSS software. Data were analyzed using descriptive and bivariate methods. Categorical variables were tested for association using chi-square analysis, whereas 1-way ANOVA was used for continuous outcomes. All statistical analyses were computed with a 95\% CI. Logistic regression was also used to examine the effects of caudal fusion level, patient age, smoking status, and biological use on reoperation rates.

\section{Results}

A total of 369 patients met criteria for inclusion. The C3-6, C3-7, and C3-T1 groups had 77, 108, and 184 patients, respectively. The average follow-up was not statistically different among groups, with a mean follow-up for C3-6, C3-7, and C3-T1 groups of 20.4, 26.6, and 27.1 months, respectively. In the $\mathrm{C} 3-7$ group, 71 patients $(65.7 \%)$ had lateral mass screws placed and 37 patients (34.3\%) had pedicle screws placed as caudal-level instrumentation. Otherwise, the C3-6 group contained all lateral mass screws and all patients in the C3-T1 group had caudal-level pedicle screws at T1. Biological use was recorded in a total of 227 patients (61.5\%). All biological use was in the form of demineralized bone matrix mixed with patient autograft.

The total reoperation rate for symptomatic pseudarthrosis or ASD was $4.8 \%$. Reoperation rates, although not significant, were lower in the C3-6 group (see Fig. 1: $2.6 \%$, vs $8.3 \%$ for $\mathrm{C} 3-7$ and $3.8 \%$ for $\mathrm{C} 3-\mathrm{T} 1 ; \mathrm{p}=0.129$ ). Causes for reoperation for each group can be seen in Fig. 2. Similarly, our rates of infection requiring revision were lower in the shorter-segment fusion without achieving statistical significance (see Fig. 3: $2.6 \%$ vs $5.6 \%$ vs $5.5 \%$ for groups 1,2 , and 3 , respectively; $\mathrm{p}=0.573$ ). See Table 1 for summary of results. Logistic regression also showed no statistical significance for age on reoperation rate $(\mathrm{p}$ $=0.485$; Table 2). By our analysis there was no statistical significance in outcomes related to reoperation for pseudarthrosis and ASD or infection rate. However, total reoperation and reoperation for pseudarthrosis neared statistical significance and may achieve significance in a higher-powered study.

Of note, blood loss and LOS for surgery were both significantly associated with caudal level of fusion segment, as seen in Fig. 4. The mean blood loss was documented as 104,125 , and $224 \mathrm{~mL}$ for groups 1,2 , and 3 , respectively ( $\mathrm{p}$ $<0.001)$. The LOSs were 4.8, 7.2, and 4.8 days ( $\mathrm{p}=0.015$; Fig. 5). Age among groups was statistically significantthe mean ages for groups 1,2 , and 3 were $68.32,61.46$, and 63.03 years, respectively $(\mathrm{p}<0.001)$. In addition, tobacco and biological use among groups was statistically different $(\mathrm{p}<0.001)$.

Age, smoking status, and biological use were recorded for each group. Logistic regression (Table 2) demonstrated that while controlling for these variables, the caudal level of fusion still lacked a statistically significant correlation 


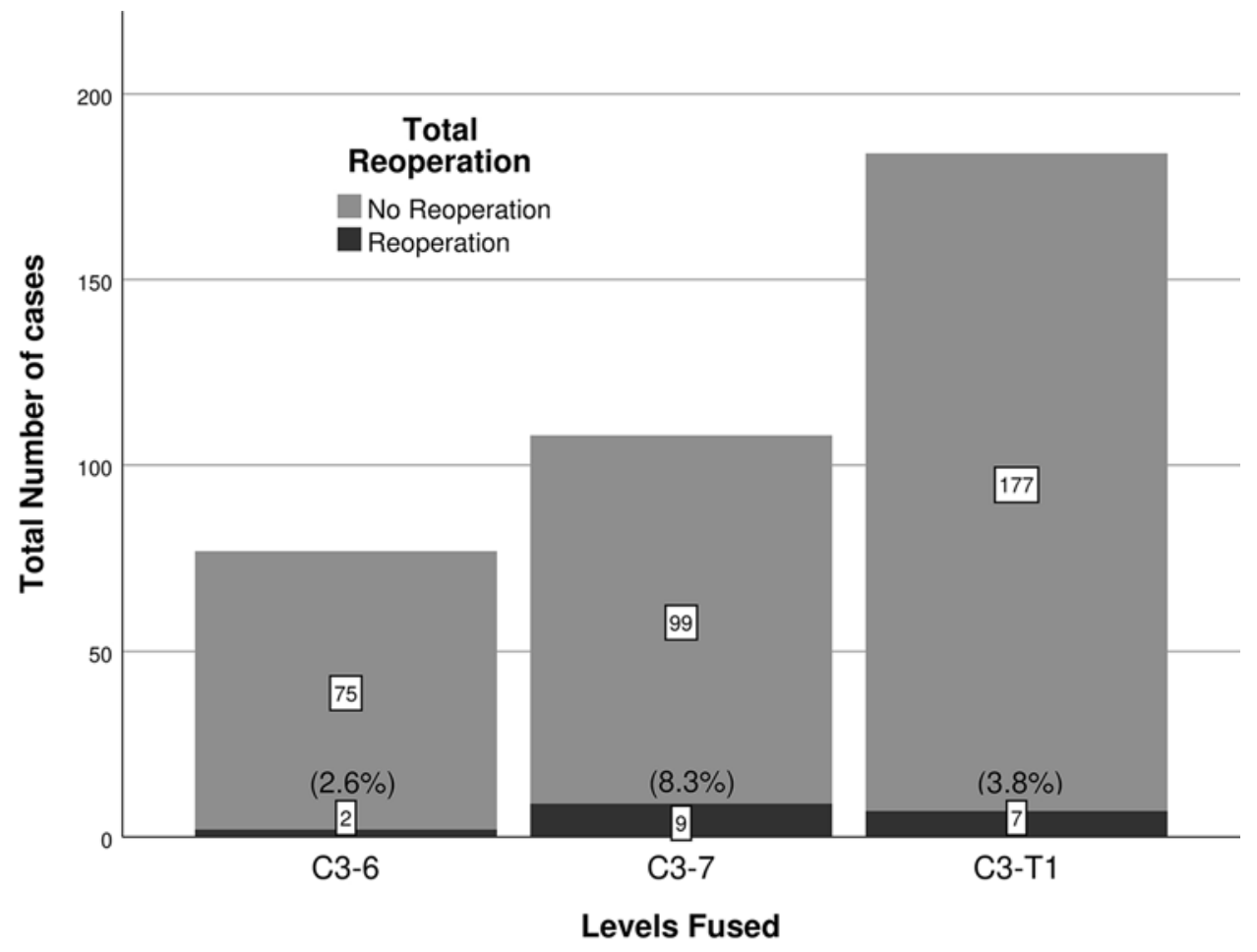

FIG. 1. Descriptive analysis demonstrating the total number and the percentage of patients who required revision surgeries by each group of fusion levels.

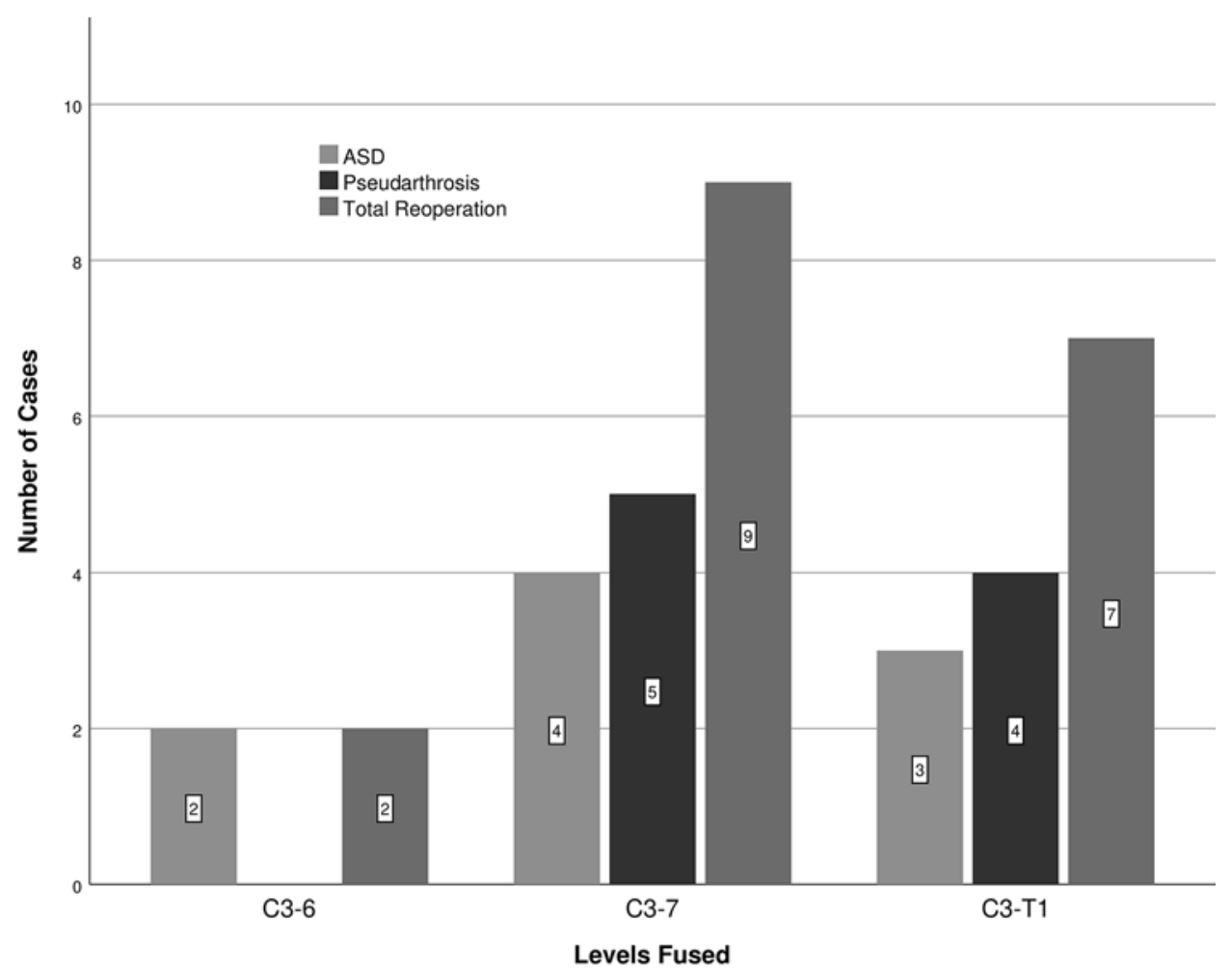

FIG. 2. Descriptive analysis demonstrating the number of reoperations by cause for each group of levels fused (excluding infection revisions). 


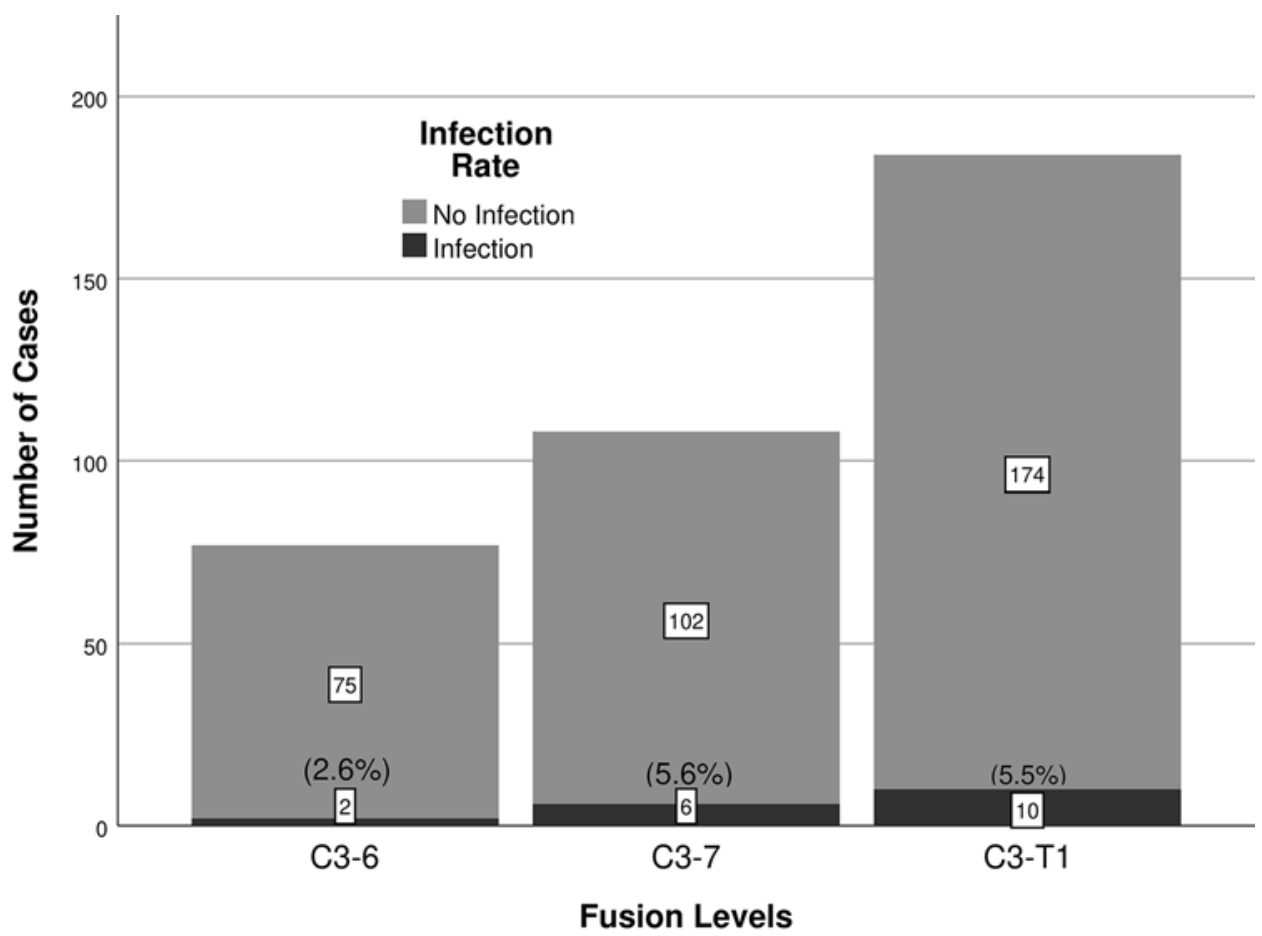

FIG. 3. Descriptive analysis demonstrating the number and the percentage of patients who required revision due to infection by each group of fusion levels. Rate of infection did not reach statistical significance.

with reoperation rates $(p=0.844)$. Of the variables, the only significant predictor of reoperation was smoking status $(\mathrm{p}=0.041)$.

Finally, ANOVA tests were run to evaluate the difference in caudal level of decompression among the fusion groups. The groups' distributions of caudal level of decompression were significantly different $(\mathrm{p}<0.001)$. Of note, $\mathrm{C} 3-7$ had $0.93 \%$ and $\mathrm{C} 3-\mathrm{T} 1$ had $0.54 \%$ of patients with a caudal level of decompression below that of the fusion construct. In contrast, the C3-6 group had $24.7 \%$ of

TABLE 1. Summary of results in 369 patients who underwent PCDF

\begin{tabular}{|c|c|c|c|c|}
\hline Factor & $C 3-6, n=77$ & $C 3-7, n=108$ & $\mathrm{C} 3-\mathrm{T} 1, \mathrm{n}=184$ & $p$ Value \\
\hline Age in yrs & $\begin{array}{c}68.32 \pm 10.91 \\
95 \% \mathrm{Cl} 65.85-70.80, \mathrm{n}=77\end{array}$ & $\begin{array}{c}61.46 \pm 11.31 \\
95 \% \mathrm{Cl} 59.26-63.66, n=104\end{array}$ & $\begin{array}{c}63.03 \pm 9.91 \\
95 \% \mathrm{Cl} 61.59-64.47, \mathrm{n}=184\end{array}$ & $<0.001^{*}$ \\
\hline Tobacco use & $15.6 \%(12)$ & $21.3 \%(23)$ & $46.2 \%(85)$ & $<0.001^{*}$ \\
\hline Biological use $†$ & $24.7 \%(19)$ & $43.5 \%(47)$ & $87.5 \%(161)$ & $<0.001^{*}$ \\
\hline Reop, no infection & $2.6 \%(2) ;$ SE $1.8 \%$ & $8.3 \%(9) ;$ SE $2.7 \%$ & $3.8 \%(7) ;$ SE1.4\% & 0.129 \\
\hline Reop for ASD & $2.6 \%(2) ;$ SE $1.8 \%$ & $3.7 \%(4) ;$ SE $1.8 \%$ & $1.6 \%$ (3); SE 0.93\% & 0.538 \\
\hline Reop for pseudarthrosis & $0 \%(0)$ & $4.6 \%$ (5); SE $2.0 \%$ & $2.2 \%(4) ;$ SE $1.1 \%$ & 0.125 \\
\hline Infection & $2.6 \%(2)$ & $5.6 \%(6)$ & $5.5 \%(10)$ & 0.573 \\
\hline Blood loss in $\mathrm{mL}$ & $\begin{array}{c}103.82 \pm 51.88 \\
95 \% \text { Cl } 86.76-120.87, n=38\end{array}$ & $\begin{array}{c}125.35 \pm 45.48 \\
95 \% \text { Cl } 114.66-136.03, n=72\end{array}$ & $\begin{array}{c}224.35 \pm 166.57 \\
95 \% \mathrm{Cl} 199.13-249.57, \mathrm{n}=170\end{array}$ & $<0.001^{*}$ \\
\hline LOS in days & $\begin{array}{c}4.77 \pm 3.55,95 \% \\
\text { Cl } 3.96-5.57, n=77\end{array}$ & $\begin{array}{c}7.18 \pm 11.97 \\
95 \% \mathrm{Cl} 4.88-9.47, n=107\end{array}$ & $\begin{array}{c}4.79 \pm 3.61 \\
95 \% \mathrm{Cl} 4.26-5.32, n=179\end{array}$ & $0.015^{*}$ \\
\hline Follow-up in mos & $\begin{array}{c}20.39 \pm 21.19,95 \% \\
\text { Cl } 15.58-25.20, n=77\end{array}$ & $\begin{array}{c}26.62 \pm 28.56 \\
95 \% \text { Cl } 21.14-32.09, n=107\end{array}$ & $\begin{array}{c}27.10 \pm 37.10 \\
95 \% \text { Cl } 15.08-39.13, n=39\end{array}$ & 0.274 \\
\hline Reop, infection included & $5.2 \%(4)$ & $13 \%(14)$ & $9.2 \%(17)$ & 0.203 \\
\hline
\end{tabular}

Values are expressed as either the mean $\pm \mathrm{SD}, 95 \% \mathrm{Cl}$, and number in subgroup —for age, blood loss, LOS, and follow-up_or as the percent (number of patients). Group 1 (C3-6), group 2 (C3-7), and group 3 (C3-T1) achieved statistically significant differences in age, tobacco use, biological use, blood loss, and LOS. Reoperation rates neared significance; however, the $\mathrm{p}$ value was $>0.05$.

* Denotes statistical significance.

$\dagger$ All biological material use was in the form of demineralized bone matrix mixed with patient autograft. 
TABLE 2. Logistic regression analysis predicting reoperation using listed variables

\begin{tabular}{lccccc}
\hline \multicolumn{1}{c}{ Variable } & B Value & SE & Significance $(\mathrm{p}$ value) & OR & $95 \% \mathrm{Cl}$ \\
\hline Level & -0.072 & 0.365 & 0.844 & 0.931 & $0.456-1.902$ \\
\hline Age & -0.015 & 0.022 & 0.485 & 0.985 & $0.944-1.028$ \\
\hline Tobacco use & -1.011 & 0.494 & $0.041^{*}$ & 0.364 & $0.138-0.959$ \\
\hline Biological use & 0.166 & 0.586 & 0.776 & 1.181 & $0.374-3.727$ \\
\hline
\end{tabular}

${ }^{*}$ Denotes significant value.

patients with caudal decompression below that of the fusion construct. These results are summarized in Table 3.

\section{Discussion}

To date, ours is the largest single-center study comparing reoperation rates for cervical spondylotic myelopathy addressed by a subaxial, posterior-only decompression and fusion. Although prior studies have advocated for extension of long-segment fusion to the thoracic spine because of lower rates of revision surgery, pseudarthrosis, or ASD,${ }^{14}$ these studies failed to distinguish between fusions ending at $\mathrm{C} 6$ and those ending at $\mathrm{C} 7$. We postulated that C6 would have less ASD and pseudarthrosis than C7 fusions because it is a shorter segment requiring fusion and preserves more segmental motion than $\mathrm{C} 7$ fusions ending at the biomechanically challenging cervicothoracic junction. ${ }^{16}$ It is also notable that our overall rates of revision surgery were consistent with sources in the literature. With an overall rate of $4.8 \%$, revision surgery after PCDF indicated for ASD or pseudarthrosis has a reported range of $3.5 \% \%^{6-8,17,18}$ to $28 \%$.

When examining variables that have had prior associations with reoperation for ASD or pseudarthrosis, linear regression analysis found no statistical difference among the fusion groups in reoperation rate despite controlling for age, tobacco use, and biological use. Given that reoperation rates for ASD and pseudarthrosis did not significantly differ, it is essential to examine the morbidity associated with a larger surgery. Patients undergoing longersegment fusions had significantly higher blood loss and LOS. Although it is not clear why the LOS was longest for C3-7, it is notable that blood loss was significantly higher and infection rates were higher in our study. If patients do not have significant degenerative disease, the morbidity of extending a fusion to the thoracic spine may outweigh any benefit with regard to stability, given the favorable outcomes with C3-6 fusions. In addition, we noted a significant relationship between treatment and age; patients who received shorter fusions tended to be older. This may reinforce the potential advantages of a shorter fusion, with similar outcomes and lower morbidity in higher-risk patients.

Last, from a quality healthcare perspective, increased lengths of fusions are also more costly in terms of medical supplies-costs can increase drastically from a 3-level fusion to a 4- or 5-level fusion. In terms of lateral mass



FIG. 4. Confidence interval demonstrating the mean of blood loss (in $\mathrm{mL}$ ) by each group of fusion levels. The means are statistically significantly different. Circles correspond to outliers and asterisks correspond to extreme outliers. 


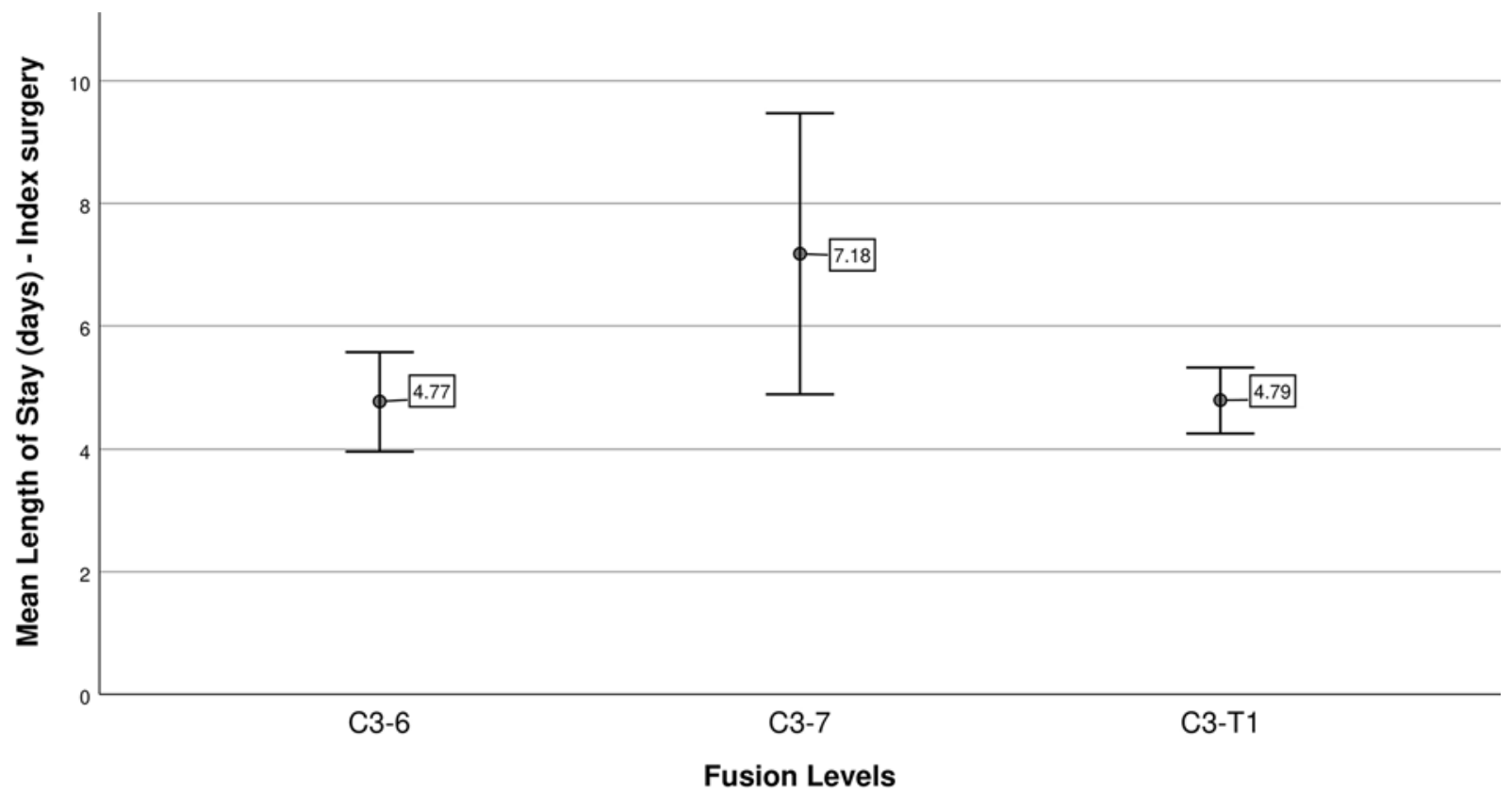

FIG. 5. Simple error bars $(95 \% \mathrm{Cl})$ demonstrating the mean LOS (in days) by each group of fusion levels. The means are statistically significantly different.

screws, rods, and caps, the cost may increase from $\$ 6260$ (3 levels) to $\$ 7550$ (4 levels) by the addition of a single level of fusion. ${ }^{19}$ In addition, pedicle screws for incorporation of thoracic levels into a fusion may cost up to $\$ 1877$ per additional level. ${ }^{20}$

Although this study provides some valuable insight into the cost and benefits of long-segment cervical fusion, it does have limitations. It is a retrospective study and patient selection did not control for levels decompressed or radiographic parameters that may have prompted a more caudal decompression and fusion. It is important to note, however, that the caudal level of decompression among the fusion groups was statistically different, as might be predicted in this retrospective analysis. Although shorter fusions may avoid a higher amount of morbidity and costly surgery, surgeons must still address the patient's pathology. The preoperative levels of compression are inherent in surgical decision-making and may preclude shorter-segment fusions in certain patients. The study was underpowered to detect the difference in reoperation rates among the groups given the lower than expected incidence. Finally, this lower incidence may be due in part to length of follow-up. On average, groups 1, 2, and 3 had a mean of 31.5, 21.1, and 36.5 months from index surgery to reoperation for pseudarthrosis or ASD. Given longer follow-up, perhaps more revision surgeries would be noted. In our study, the mean follow-up was shorter than the average time to reoperation for groups 1 and 3. This could be indicative of a lower failure rate, or a slower time to failure than in group 2. However, longer follow-up is necessary in the future to adequately evaluate a late endpoint such as ASD.

\section{Conclusions}

Previous literature has advocated for the extension of cervical fusion past the cervicothoracic junction to decrease pseudarthrosis and ASD by fusion to rigid spinal anatomy. However, this literature often fails to examine the potential benefits of leaving an additional mobile segment by ending the fusion at C6. This study's results demonstrate no statistical difference in reoperation rates for long-segment cervical fusions ending at C6, C7, or T1. These results are reassuring to surgeons who wish to avoid the morbidity and cost associated with a longer fusion in high-risk surgical candidates or elderly patients.

TABLE 3. ANOVA results regarding fusion versus caudal level of decompression

\begin{tabular}{ccccccc}
\hline & \multicolumn{5}{c}{ Caudal Level } & \multirow{2}{*}{ Total } \\
\cline { 2 - 6 } Group & C5 & C6 & C7 & T1 & T2 & $77(100 \%)$ \\
\hline C3-6 & $3(3.90 \%)$ & $55(71.42 \%)$ & $18(23.38 \%)$ & $1(1.30 \%)$ & $0(0 \%)$ & $108(100 \%)$ \\
\hline C3-7 & $1(0.93 \%)$ & $19(17.59 \%)$ & $86(79.63 \%)$ & $1(0.93 \%)$ & $1(0.93 \%)$ & $184(100 \%)$ \\
\hline C3-T1 & $1(0.54 \%)$ & $17(9.24 \%)$ & $123(66.85 \%)$ & $42(22.83 \%)$ & $1(0.54 \%)$ & 184 \\
\hline$p<0.001$, indicating a significant difference in distribution of caudal level of decompression among fusion groups.
\end{tabular}




\section{References}

1. Fehlings MG, Barry S, Kopjar B, et al. Anterior versus posterior surgical approaches to treat cervical spondylotic myelopathy: outcomes of the prospective multicenter AOSpine North America CSM study in 264 patients. Spine (Phila Pa 1976). 2013;38(26):2247-2252.

2. Youssef JA, Heiner AD, Montgomery JR, et al. Outcomes of posterior cervical fusion and decompression: a systematic review and meta-analysis. Spine J. 2019;19(10):1714-1729.

3. Liu CY, Zygourakis CC, Yoon S, et al. Trends in utilization and cost of cervical spine surgery using the National Inpatient Sample database, 2001 to 2013. Spine (Phila Pa 1976). 2017;42(15):E906-E913.

4. Vonck CE, Tanenbaum JE, Smith GA, et al. National trends in demographics and outcomes following cervical fusion for cervical spondylotic myelopathy. Global Spine J. 2018;8(3): 244-253.

5. Cho SK, Riew KD. Adjacent segment disease following cervical spine surgery. J Am Acad Orthop Surg. 2013;21(1):3-11.

6. Schroeder GD, Kepler CK, Kurd MF, et al. Is it necessary to extend a multilevel posterior cervical decompression and fusion to the upper thoracic spine? Spine (Phila Pa 1976). 2016, 41(23):1845-1849.

7. Truumees E, Singh D, Geck MJ, Stokes JK. Should long-segment cervical fusions be routinely carried into the thoracic spine? A multicenter analysis. Spine J. 2018;18(5):782-787.

8. Auerbach J, Cho W, Sehn J, et al. Crossing the cervico-thoracic junction in long posterior cervical fusions reduces the rate of symptomatic adjacent segment breakdown. Paper presented at: NASS 26th Annual Meeting; November 2, 2011; Chicago, IL.

9. Badiee RK, Mayer R, Pennicooke B, et al. Complications following posterior cervical decompression and fusion: a review of incidence, risk factors, and prevention strategies. J Spine Surg. 2020;6(1):323-333.

10. Lee DH, Cho JH, Jung JI, et al. Does stopping at C7 in long posterior cervical fusion accelerate the symptomatic breakdown of cervicothoracic junction? PLoS One. 2019;14(5): e0217792.

11. Yang JS, Buchowski JM, Verma V. Construct type and risk factors for pseudarthrosis at the cervicothoracic junction. Spine (Phila Pa 1976). 2015;40(11):E613-E617.

12. Hashimoto K, Aizawa T, Kanno H, Itoi E. Adjacent segment degeneration after fusion spinal surgery-a systematic review. Int Orthop. 2019;43(4):987-993.

13. Goyal A, Akhras A, Wahood W, et al. Should multilevel posterior cervical fusions involving $\mathrm{C} 7$ cross the cervicothoracic junction? A systematic review and meta-analysis. World Neurosurg. 2019;127:588-595.e5.

14. Chan AK, Badiee RK, Rivera J, et al. Crossing the cervicothoracic junction during posterior cervical fusion for myelopathy is associated with superior radiographic parameters but similar clinical outcomes. Neurosurgery. 2020;87(5): 1016-1024.
15. Fayed I, Toscano DT, Triano MJ, et al. Crossing the cervicothoracic junction during posterior cervical decompression and fusion: is it necessary? Neurosurgery. 2020;86(6):E544E550.

16. Steinmetz MP, Miller J, Warbel A, et al. Regional instability following cervicothoracic junction surgery. J Neurosurg Spine. 2006;4(4):278-284.

17. Woodroffe RW, Helland LC, Grossbach AJ, et al. Risk factors associated with reoperation in posterior cervical fusions: a large-scale retrospective analysis. Clin Neurol Neurosurg. 2020;195:105828.

18. Xia Y, Xu R, Kosztowski TA, et al. Reoperation for proximal adjacent segment pathology in posterior cervical fusion constructs that fuse to C2 vs C3. Neurosurgery. 2019;85(3): E520-E526.

19. Ray WZ, Ravindra VM, Jost GF, et al. Cost effectiveness of subaxial fusion-lateral mass screws versus transarticular facet screws. Neurosurg Focus. 2012;33(1):E14.

20. Jaquith BP, Chase A, Flinn P, et al. Screws versus hooks: implant cost and deformity correction in adolescent idiopathic scoliosis. J Child Orthop. 2012;6(2):137-143.

\section{Disclosures}

Dr. Schroeder is a consultant for Stryker, Zimmer, Astura, Medtronic, Teledoc, WK, AO Spine, and Bioventus.

\section{Author Contributions}

Conception and design: Hines, Wilt, Mahtabfar, Elmer, Gonzalez, Montenegro, Patel, Detweiler, Fatema, Schroeder, Harrop. Acquisition of data: Hines, Franco, Mahtabfar, Elmer, Gonzalez, Montenegro, Velagapudi, Patel, Detweiler, Fatema, Schroeder. Analysis and interpretation of data: Hines, Franco, Mahtabfar, Elmer, Gonzalez, Montenegro, Velagapudi, Patel, Detweiler, Fatema, Schroeder, Harrop. Drafting the article: Hines, Wilt, Franco, Elmer, Gonzalez, Montenegro, Detweiler, Fatema, Harrop. Critically revising the article: Hines, Wilt, Schroeder, Harrop. Reviewed submitted version of manuscript: Hines. Approved the final version of the manuscript on behalf of all authors: Hines. Statistical analysis: Velagapudi, Patel, Harrop. Study supervision: Schroeder, Harrop.

\section{Correspondence}

Kevin Hines: Thomas Jefferson University Hospital, Philadelphia, PA.kevin.hines@jefferson.edu. 